\title{
The Linear Algebra of UTP
}

\author{
Bernhard Möller \\ Institut für Informatik, Universität Augsburg, D-86135 Augsburg, Germany \\ moeller@informatik. uni-augsburg. de
}

\begin{abstract}
We show that the well-known algebra of matrices over a semiring can be used to reason conveniently about predicates as used in the Unifying Theories of Programming (UTP). This allows a simplified treatment of the designs of Hoare and He and the prescriptions of Dunne. In addition we connect the matrix approach with the theory of test and condition semirings and the modal operators diamond and box. This allows direct re-use of the results and proof techniques of Kleene algebra with tests for UTP as well as a connection to traditional wp/wlp semantics. Finally, we show that matrices of predicate transformers allow an even more streamlined treatment and removal of a restricting assumption on the underlying semirings.
\end{abstract}

\section{Introduction}

In the Unifying Theories of Programming (UTP) [5] the termination behaviour of programs is modelled using two special variables $o k$ and $o k^{\prime}$ that express whether a program has been started and has terminated, respectively. Programs are identified with predicates that relate the initial values $v$ of variables with their final values $v^{\prime}$; moreover, $o k$ and $o k^{\prime}$ may occur freely in such predicates.

The aim of the present paper is to present a calculationally more workable form of the theory of predicates and designs that does no longer mention the "unobservable" variables $o k$ and $o k^{\prime}$; in fact it is even completely variable-free and hence, in particular, does not need to work with substitutions. This makes calculations not only simpler, but also safer. Truly hiding the unobservables is important, since their unchecked use can lead to inconsistencies and paradoxes such as the Dead Variable Paradox.

The remainder of this paper is organised as follows. Section 2 presents the basic idea of the matrix model of UTP predicates, while Section 3 deals with the special predicate class of UTP designs. In Section 4 we abstract from the concrete case of predicates over program variables to that of matrices over semirings; next to greater generality this yields a more compact notation. In Section 5 designs are discussed in this more general setting, while Section 6 gives an algebraic formulation of the healthiness conditions that distinguish designs. Designs were introduced to model total correctness, in particular, non-miraculous programs. Section 7 deals with another subclass of UTP predicates, the prescriptions, that model the view of general correctness and may show miraculous behaviour. Section 8 contains a more detailed treatment of conditions, i.e., predicates that only 
depend on input values of variables, and the related concept of tests in semirings. This allows direct re-use of the results and proof techniques of Kleene algebra with tests for UTP. Sections 9 and 10 employ tests and conditions to establish the link with traditional wlp/wp semantics. However, at a certain point the connection is not as smooth as one may wish. This is remedied in Section 11 using "higher-level" predicate transformers. Section 12 presents a brief conclusion.

To keep the overall structure of the paper clearer, some technicalities are deferred an appendix.

\section{A Matrix View of UTP}

Our main aim is to get rid of explicit uses of the special variables $o k$ and $o k^{\prime}$. We achieve this by recording, for each combination of possible values of these two variables, the residual predicate that depends only on the proper program variables. To emphasise the dependence of a general UTP predicate on $o k$ and $o k^{\prime}$ we use the notation $R\left(o k, o k^{\prime}\right)$. The basic idea of our matrix calculus is now to represent $R$ by a $2 \times 2$-matrix. The rows are indexed by the values of $o k$ and the columns by those of $o k^{\prime}$; the entries are the residual predicates in which $o k$ and $o k^{\prime}$ do not occur, i.e.,

$$
R=\left(\begin{array}{ll}
R(\text { false }, \text { false }) & R(\text { false }, \text { true }) \\
R(\text { true }, \text { false }) & R(\text { true }, \text { true })
\end{array}\right) .
$$

In this view, a predicate $P$ not depending on $o k$ and $o k^{\prime}$ corresponds to the constant matrix

$$
\left(\begin{array}{ll}
P & P \\
P & P
\end{array}\right)
$$

The matrix representation may seem a complication at first. But let us look at sequential composition of UTP predicates, defined as

$$
R ; S \Leftrightarrow{ }_{d f} \exists o k_{0}, v_{0}: R\left[o k_{0}, v_{0} / o k^{\prime}, v^{\prime}\right] \wedge S\left[o k_{0}, v_{0} / o k, v\right],
$$

where $v$ (also with index or prime) stands for the list of all proper program variables. We emphasise again the dependence on $o k$ and $o k^{\prime}$. To this end we also split the existential quantifier into the parts concerning the unobservables and the proper variables; afterwards the proper part can be folded into a composition of its own:

$$
\begin{aligned}
(R ; S)\left(o k, o k^{\prime}\right) & \Leftrightarrow d f \exists o k_{0}: \exists v_{0}: R\left(o k, o k_{0}\right)\left[v_{0} / v^{\prime}\right] \wedge S\left(o k_{0}, o k^{\prime}\right)\left[v_{0} / v\right] \\
& \Leftrightarrow \quad \exists o k_{0}: R\left(o k, o k_{0}\right) ; S\left(o k_{0}, o k^{\prime}\right) .
\end{aligned}
$$

This now has a convenient matrix interpretation. As in graph algorithms such as Warshall's, we can view $\exists o k_{0}$ as summation over all possible values of $o k_{0}$ and ; as elementwise multiplication. With this interpretation the above formula gives just the entries for the product of the matrices $R$ and $S$, i.e., $R ; S=R \cdot S$. 
The advantage of this view is that composition can now be treated in a completely component-free manner and existential quantification and substitution disappear. Moreover, the pseudo-variables $o k$ and $o k^{\prime}$ need no longer be mentioned explicitly at all. If for some reason we need to reason about them explicitly, we can represent them as

$$
o k=\left(\begin{array}{cc}
\text { false } & \text { false } \\
\text { true } & \text { true }
\end{array}\right), \quad o k^{\prime}=\left(\begin{array}{cc}
\text { false } & \text { true } \\
\text { false } & \text { true }
\end{array}\right) .
$$

Next to composition, the matrix algebra supports the Boolean operations: negation, conjunction and disjunction all are defined componentwise. Setting $R \Rightarrow S \Leftrightarrow{ }_{d f} \quad R \vee S=S$, also implication works componentwise.

\section{Designs}

As a subclass of the general UTP predicates, Hoare and He introduce designs, that reflect an assumption/commitment style of specification, of the form

$$
P \vdash Q \Leftrightarrow_{d f} \text { ok } \wedge P \Rightarrow o k^{\prime} \wedge Q
$$

where $o k$ and $o k^{\prime}$ are not allowed to occur in $P$ or $Q$. The informal meaning is: if a computation allowed by the design has started in a state that satisfies the precondition $P$ it will eventually terminate in a state that satisfies the postcondition $Q$. By plugging in the possible combinations of the values of $o k$ and $o k^{\prime}$ we obtain the matrix representation

$$
P \vdash Q=\left(\begin{array}{cc}
\text { true } & \text { true } \\
\bar{P} & \bar{P} \vee Q
\end{array}\right)
$$

To show a first example of the matrix calculus at work, let us derive this representation algebraically:

$$
\begin{aligned}
& \text { ok } \wedge P \Rightarrow \text { ok } \wedge Q \\
= & \left(\begin{array}{cc}
\text { false } & \text { false } \\
\text { true } & \text { true }
\end{array}\right) \wedge\left(\begin{array}{cc}
P & P \\
P & P
\end{array}\right) \Rightarrow\left(\begin{array}{cc}
\text { false } & \text { true } \\
\text { false } & \text { true }
\end{array}\right) \wedge\left(\begin{array}{ll}
Q & Q \\
Q & Q
\end{array}\right) \\
= & \left(\begin{array}{cc}
\text { false } & \text { false } \\
P & P
\end{array}\right) \vee\left(\begin{array}{cc}
\text { false } & Q \\
\text { false } & Q
\end{array}\right) \\
= & \left(\begin{array}{cc}
\text { true } & \text { true } \\
\bar{P} & \bar{P}
\end{array}\right) \vee\left(\begin{array}{cc}
\text { false } & Q \\
\text { false } & Q
\end{array}\right) \\
= & \left(\begin{array}{cc}
\text { true } & \text { true } \\
\bar{P} & \bar{P} \vee Q
\end{array}\right) .
\end{aligned}
$$

We defer further calculations till we obtain a more compact notation in the next section. 


\section{Abstracting to Semirings}

Again, as in certain graph algorithms, it is useful to base the treatment not on the concrete model of matrices over predicates but on matrices over semirings. Semirings provide the basic operations of choice and sequential composition under the notations + and - as well as a basic set of algebraic laws for these. A weak semiring is a structure $(S,+, \cdot, 0,1)$ such that

$-(S,+, 0)$ is a commutative monoid,

$-(S, \cdot, 1)$ is a monoid,

- operation $\cdot$ distributes over + in both arguments

- and 0 is a left annihilator, i.e., $0 \cdot a=0$.

A semiring is a weak semiring in which 0 is also a right annihilator, i.e., $a \cdot 0=0$. Sometimes for emphasis we write "full semiring" instead of just "semiring".

A (weak) semiring is idempotent if + is idempotent, i.e., $a+a=a$. In this case the relation $a \leq b \Leftrightarrow_{d f} a+b=b$ is a partial order, called the natural order on $S$. It has 0 as its least element. Moreover, + and $\cdot$ are isotone w.r.t. $\leq$ and $a+b$ is the least upper bound or join of $a$ and $b$ w.r.t. $\leq$.

A (weak) idempotent semiring is Boolean if it also has a greatest-lower-bound or meet operation $\wedge$, such that + and $\wedge$ distribute over each other, and a complement operation - that satisfies de Morgan's laws as well as $a \wedge \bar{a}=0$ and $a+\bar{a}=\top$, where $\top=\overline{0}$ is the greatest element. In other words, a Boolean semiring is a Boolean algebra with a sequential composition operation. To save parentheses we use the convention that $\wedge$ binds tighter than + but equally tight as · does. We will freely use the implication operator $a \rightarrow b={ }_{d f} \bar{a}+b$ and its standard laws. We use $\wedge$ rather than $\sqcap$ for the meet to avoid a clash of notation between semiring theory and the theory of UTP. To disambiguate the formulas we use a larger $\wedge$ for meta-logical conjunction.

An important property is multiplicative idempotence of $T$ :

$$
\top \cdot \top=\top .
$$

The direction $(\leq)$ is trivial, since $\top$ is the greatest element. The converse direction follows by neutrality and isotonicity: $\top=\top \cdot 1 \leq \top \cdot \top$.

From now on we assume $S$ to be a full idempotent Boolean semiring.

In the previous section we have already used the Boolean semiring of UTP predicates with ; as composition. Another important semiring is REL(M), the algebra of binary relations under union and composition over a set $M$, of which the predicates form a special instance.

Many other examples exist but will not be used here except for the matrix semiring. Let $(S,+, \cdot, 0,1)$ be a semiring and $M$ be a finite set. Then the set $S^{M \times M}$ of functions from $M \times M$ to $S$ can be viewed as the set of $|M| \times|M|$ matrices with indices in $M$ and elements in $S$. Consider the structure $\operatorname{MAT}(M, S)=\left(S^{M \times M},+, \cdot, \mathbf{0}, \mathbf{1}\right)$ where + and $\cdot$ are the usual operations of matrix addition and multiplication, and $\mathbf{0}$ and $\mathbf{1}$ are the zero and unit matrices. Then $\operatorname{MAT}(M, S)$ again forms a semiring, the matrix semiring over $M$ and $S$. 
$\operatorname{MAT}(M, S)$ is idempotent if $S$ is. In this case, the natural order is the componentwise one. If $S$ is Boolean, so is $\operatorname{MAT}(M, S)$, with componentwise meet.

Taking $S$ to be the two-element Boolean semiring of truth values yields the usual Boolean matrix representation of $\operatorname{REL}(\mathrm{M})$ as $\operatorname{MAT}(M, S)$ in terms of adjacency matrices.

For abstractly representing predicates that depend on two Boolean variables $o k$ and $o k^{\prime}$ we use $2 \times 2$-matrices with elements from a Boolean semiring $S$ as entries. The element 1 represents the predicate $\operatorname{skip} \Leftrightarrow d f \quad v=v^{\prime}$. We will use the identifiers false, skip and true instead of 0,1 and $T$ when appropriate.

For convenience we define

$$
o k={ }_{d f}\left(\begin{array}{cc}
0 & 0 \\
\top & \top
\end{array}\right), \quad o k^{\prime}=_{d f}\left(\begin{array}{cc}
0 & \top \\
0 & \top
\end{array}\right) .
$$

\section{The Algebra of Designs}

Generalising formula (1), we set for elements $a, b \in S$ of a Boolean semiring $S$

$$
a \vdash b={ }_{d f}\left(\begin{array}{cc}
\top & \top \\
\bar{a} & \bar{a}+b
\end{array}\right),
$$

with + now playing the role of disjunction or choice.

We want to calculate the behaviour of designs under + and $\cdot$. First,

$$
\begin{aligned}
& (a \vdash b)+(c \vdash d)=\left(\begin{array}{cc}
\top & \top \\
\bar{a} & \bar{a}+b
\end{array}\right)+\left(\begin{array}{cc}
\top & \top \\
\bar{c} & \bar{c}+d
\end{array}\right)= \\
& \left(\begin{array}{cc}
\top & \top \\
\bar{a}+\bar{c} & \bar{a}+b+\bar{c}+d
\end{array}\right)=\left(\begin{array}{cc}
\frac{T}{(c \wedge a)} & \overline{(c \wedge a)}+b+d
\end{array}\right)=(c \wedge a) \vdash(b+d) .
\end{aligned}
$$

In particular, the design $T \vdash 0$, which is the same as $\overline{o k}$, is a neutral element w.r.t. + . Moreover, we obtain

$$
(a \vdash b) \leq(c \vdash d) \Leftrightarrow(a \vdash b)+(c \vdash d)=(c \vdash d) \Leftrightarrow(c \leq a) \wedge(c \wedge b \leq d)
$$

and

$$
(a \vdash b)=(c \vdash d) \Leftrightarrow a=c \wedge \bar{a}+b=\bar{c}+d \Leftrightarrow a=c \wedge(a \wedge b=c \wedge d) .
$$

For composition we obtain, using (2),

$$
\begin{aligned}
& (a \vdash b) \cdot(c \vdash d) \\
& =\left(\begin{array}{cc}
\top & \top \\
\bar{a} & \bar{a}+b
\end{array}\right) \cdot\left(\begin{array}{cc}
\top & \top \\
\bar{c} & \bar{c}+d
\end{array}\right) \\
& =\left(\begin{array}{cc}
\top \cdot \top+\top \cdot \bar{c} & \top \cdot \top+\top \cdot(\bar{c}+d) \\
\bar{a} \cdot \top+(\bar{a}+b) \cdot \bar{c} & \bar{a} \cdot \top+(\bar{a}+b) \cdot(\bar{c}+d)
\end{array}\right) \\
& =\left(\begin{array}{cc}
T & \\
\bar{a} \cdot \top+\bar{a} \cdot \bar{c}+b \cdot \bar{c} & \bar{a} \cdot \top+\bar{a} \cdot(\bar{c}+d)+b \cdot \bar{c}+b \cdot d
\end{array}\right)
\end{aligned}
$$




$$
\begin{aligned}
& =\left(\begin{array}{cc}
\top & \top \\
\bar{a} \cdot \top+b \cdot \bar{c} & \bar{a} \cdot \top+b \cdot \bar{c}+b \cdot d
\end{array}\right) \\
& =\left(\frac{T}{\overline{\bar{a} \cdot \top} \wedge \overline{b \cdot \bar{c}}} \frac{T}{\overline{\bar{a} \cdot \top \wedge \overline{b \cdot \bar{c}}}+b \cdot d}\right) \\
& =(\overline{\bar{a} \cdot \top} \wedge \overline{b \cdot \bar{c}}) \vdash(b \cdot d) \text {. }
\end{aligned}
$$

Summarised,

$$
(a \vdash b) \cdot(c \vdash d)=(\overline{\bar{a} \cdot \top} \wedge \overline{b \cdot \bar{c}}) \vdash(b \cdot d) .
$$

In particular, within the set of designs both $\overline{o k}=\top \vdash 0$ and true $=_{d f}\left(\begin{array}{cc}T & \top \\ \top & \top\end{array}\right)$ are left zeros and $\mathbb{I}=_{d f} \top \vdash 1$ is a left-neutral element w.r.t. composition.

\section{Healthiness Conditions}

In [5] the UTP predicates are classified according to certain healthiness conditions. In matrix terminology, designs are characterised by two properties:

(H1) The first row must be constantly $T$.

(H2) Both rows must be increasing w.r.t $\leq$.

Clearly every design of the form (3) satisfies (H1) and (H2). Conversely, if $a \leq b$ then $\left(\begin{array}{cc}\top & \top \\ a & b\end{array}\right)=\left(\begin{array}{cc}\top & \top \\ a & a+b\end{array}\right)=\bar{a} \vdash b$, so that $\left(\begin{array}{cc}\top & \top \\ a & b\end{array}\right)$ is a design.

Clearly, matrix $A$ satisfies (H1) iff $A=\left(\begin{array}{cc}\top & \top \\ 0 & 0\end{array}\right)+A=o k \rightarrow A$ (see also Theorem 3.1.4 in [5]).

This type of characterisation by a fixpoint property is particularly useful if the underlying Boolean semiring (and hence the matrix semiring over it) is even a complete lattice, since Tarski's fixpoint theorem then implies that the set of all (H1) predicates forms a complete sublattice.

Next we show how the fixpoint characterisation of (H2) given in Example 4.1.21(1) of [5] can be derived in a systematic way in our matrix calculus. First we observe that

$$
\left(\begin{array}{ll}
a & b \\
c & d
\end{array}\right) \text { satisfies }(\mathrm{H} 2) \Leftrightarrow a+b=b \wedge c+d=d \Leftrightarrow\left(\begin{array}{ll}
a & b \\
c & d
\end{array}\right)=\left(\begin{array}{ll}
a & a+b \\
c & c+d
\end{array}\right) .
$$

So if we manage to generate the latter matrix from the original one by an isotone function defined in terms of the algebra we are done.

In linear algebra this type of transformation is known as a shearing and can be described by the multiplication

$$
\left(\begin{array}{ll}
a & a+b \\
c & c+d
\end{array}\right)=\left(\begin{array}{ll}
a & b \\
c & d
\end{array}\right) \cdot\left(\begin{array}{ll}
1 & 1 \\
0 & 1
\end{array}\right)
$$

The shearing matrix can be decomposed as follows:

$$
\left(\begin{array}{ll}
1 & 1 \\
0 & 1
\end{array}\right)=\left(\begin{array}{ll}
\top & \top \\
0 & \top
\end{array}\right) \wedge\left(\begin{array}{ll}
1 & 1 \\
1 & 1
\end{array}\right)=(\top \vdash \top) \wedge\left(\begin{array}{ll}
1 & 1 \\
1 & 1
\end{array}\right)
$$

Therefore we have the following result. 
Lemma 6.1 A satisfies (H2) iff $A=A \cdot B$ where

$$
B=(\top \vdash \top) \wedge\left(\begin{array}{ll}
1 & 1 \\
1 & 1
\end{array}\right)
$$

This is indeed a fixpoint characterisation with an isotone generating function, and so the set of all (H2)-matrices forms a complete lattice (provided the underlying semiring $S$ is complete).

The further healthiness conditions (H3) and (H4) serve to characterise the designs for which $T \vdash 0$ and $T \vdash 1$ are also a right zero and a right-neutral element w.r.t. ', respectively. They are directly given as algebraic conditions:

(H3) $A \cdot \mathbb{I}=A$.

(H4) $A \cdot$ true $=$ true.

By distributivity and associativity it is immediate that each of the classes (H3) and (H4) is closed under addition and composition.

We only work these properties out for the case where $A$ is a design. Here it is easier to work directly with the matrices than going through the composition formula for designs. For (H3) we calculate

$$
(a \vdash b) \cdot \mathbb{I}=\left(\begin{array}{cc}
\top & \top \\
\bar{a} & \bar{a}+b
\end{array}\right) \cdot\left(\begin{array}{cc}
\top & \top \\
0 & 1
\end{array}\right)=\left(\begin{array}{cc}
\top & \top \\
\bar{a} \cdot \top & \bar{a} \cdot \top+\bar{a}+b
\end{array}\right),
$$

so that $a \vdash b$ satisfies (H3) iff $\bar{a} \cdot \top=\bar{a} \Leftrightarrow \bar{a} \cdot \top \leq \bar{a}$.

This means that $\bar{a}$ has to be a right ideal (in UTP also known as a condition). In the semiring REL of relations this is equivalent to $a$ itself being a right ideal, since by Schröder's law

$$
\bar{a} \cdot \top \leq \bar{a} \Leftrightarrow a \cdot \top^{\cup} \leq a \Leftrightarrow a \cdot \top \leq a .
$$

In general semirings this need not be the case.

Following [3], we call matrices satisfying (H3) normal. For normal designs we obtain the simplified composition formula (see also Theorem 3.2.4 in [5])

$$
(a \vdash b) \cdot(c \vdash d)=(a \wedge \overline{b \cdot \bar{c}}) \vdash(b \cdot d) .
$$

Various authors have noticed that (H3) implies (H2). In matrix algebra this can be verified as follows:

$$
\left(\begin{array}{ll}
a & b \\
c & d
\end{array}\right) \cdot \mathbb{I}=\left(\begin{array}{ll}
a \cdot \top & a \cdot T+b \\
c \cdot \top & c \cdot \top+d
\end{array}\right)
$$

The matrix on the right-hand side clearly is (H2). So if $A$ is (H3), i.e., if $A=A \cdot \mathbb{I I}$, it is also (H2).

For (H4) we calculate

$$
(a \vdash b) \cdot \text { true }=\left(\begin{array}{cc}
\top & \top \\
\bar{a} & \bar{a}+b
\end{array}\right) \cdot\left(\begin{array}{cc}
\top & \top \\
\top & \top
\end{array}\right)=\left(\begin{array}{cc}
\top & \top \\
(\bar{a}+b) \cdot \top & (\bar{a}+b) \cdot \top
\end{array}\right),
$$


so that $a \vdash b$ satisfies (H4) iff

$$
(\bar{a}+b) \cdot \top=\top .
$$

Matrices satisfying (H4) are called feasible in [5].

Let $\operatorname{ND}(S)$ be the set of all normal designs over $S$. Collecting the stated algebraic properties, we obtain

Lemma 6.2 The structure $(\operatorname{ND}(S),+, \cdot, \overline{o k}, \mathbb{I})$ is a weak semiring.

There is no analogous result for the feasible normal designs, since there is not even a neutral element w.r.t. addition. This can be shown as follows. A neutral element $t \vdash a$ would have to be least w.r.t. the natural semiring order, i.e., would need to satisfy, for all $s, t$,

$$
t \vdash a \leq s \vdash b \Leftrightarrow s \leq t \wedge s \wedge a \leq b .
$$

Setting $s=\top$ we obtain $t=\top$ and the residual requirement that $a \leq b$ for all $b$. So the only candidate would be $T \vdash 0=\overline{o k}$ which, however, is not feasible.

\section{The Algebra of Prescriptions}

Whereas (feasible) designs reflect the semantic view of total correctness, in [3] Dunne models the view of general correctness as introduced in [11-13] that also allows miraculous program behaviour. To this end Dunne introduces prescriptions of the form

$$
P \Vdash Q \quad \Leftrightarrow_{d f} \quad\left(o k \wedge P \Rightarrow o k^{\prime}\right) \wedge\left(o k^{\prime} \Rightarrow Q \wedge o k\right) .
$$

By investigating the four possible combinations of the values of $o k$ and $o k^{\prime}$, or by a calculation analogous to the one for designs in Section 2, one obtains the matrix representation

$$
P \Vdash Q \Leftrightarrow\left(\begin{array}{cc}
\text { true } & \text { false } \\
\bar{P} & Q
\end{array}\right) .
$$

Since the first row of our matrices corresponds to the case $o k=$ false, this yields immediately Dunne's healthiness condition $(\underline{\mathrm{HP}})$ : A matrix $A$ represents a prescription iff its first row coincides with that of $\overline{o k^{\prime}}$.

The generalisation to Boolean semirings reads

$$
a \Vdash b={ }_{d f}\left(\begin{array}{cc}
\top & 0 \\
\bar{a} & b
\end{array}\right) .
$$

From this it is immediate that, unlike designs, prescriptions can uniquely be decomposed into their constituents:

$$
(a \Vdash b)=(c \Vdash d) \Leftrightarrow a=c \wedge b=d .
$$


Moreover, since the natural order on matrices works componentwise,

$$
(a \Vdash b) \leq(c \Vdash d) \Leftrightarrow c \leq a \wedge b \leq d .
$$

Let us now see how addition and composition of prescriptions work out. First,

$$
(a \Vdash b)+(c \Vdash d)=\left(\begin{array}{cc}
\top & 0 \\
\bar{a} & b
\end{array}\right)+\left(\begin{array}{cc}
\top & 0 \\
\bar{c} & d
\end{array}\right)=\left(\begin{array}{cc}
\top & 0 \\
\overline{a \wedge c} & b+d
\end{array}\right)=a \wedge c \Vdash b+d .
$$

Second, since we assume $S$ to be a full semiring,

$$
\begin{aligned}
& (a \Vdash b) \cdot(c \Vdash d)=\left(\begin{array}{cc}
\top & 0 \\
\bar{a} & b
\end{array}\right) \cdot\left(\begin{array}{cc}
\top & 0 \\
\bar{c} & d
\end{array}\right)=\left(\begin{array}{cc}
\top \cdot T+0 \cdot \bar{c} & \top \cdot 0+0 \cdot d \\
\bar{a} \cdot \top+b \cdot \bar{c} & \bar{a} \cdot 0+b \cdot d
\end{array}\right) \\
& =\left(\begin{array}{cc}
\top & 0 \\
\overline{\bar{a} \cdot \top} \wedge \overline{b \cdot \bar{c}} & b \cdot d
\end{array}\right)=(\overline{\bar{a} \cdot \top} \wedge \overline{b \cdot \bar{c}}) \Vdash(b \cdot d) .
\end{aligned}
$$

Summarised,

$$
(a \Vdash b) \cdot(c \Vdash d)=(\overline{\bar{a} \cdot \bar{T}} \wedge \overline{b \cdot \bar{c}}) \Vdash(b \cdot d) .
$$

So, in particular, the set of prescriptions is closed under choice and composition. The formulas for addition and composition coincide with the ones for designs.

The following prescriptions are of particular importance (see Dunne [3] and Nelson [13]):

$$
\begin{array}{rl}
\text { loop }=_{d f} & 0 H 0=\left(\begin{array}{cc}
\top & 0 \\
\top & 0
\end{array}\right)=\overline{o k^{\prime}}, \\
\text { fail }=_{d f} & \top H 0=\left(\begin{array}{cc}
\top & 0 \\
0 & 0
\end{array}\right)=\overline{o k} \wedge \overline{o k^{\prime}}, \\
\text { chaos }=_{d f} & 0 H \top=\left(\begin{array}{cc}
\top & 0 \\
\top & \top
\end{array}\right)=o k^{\prime} \rightarrow o k, \\
\text { havoc }=_{d f} \quad T H \top=\left(\begin{array}{cc}
\top & 0 \\
0 & T
\end{array}\right)=o k \leftrightarrow o k^{\prime}, \\
\text { skip }=_{d f} \quad T H 1=\left(\begin{array}{cc}
\top & 0 \\
0 & 1
\end{array}\right) .
\end{array}
$$

Since the composition rule for prescriptions is the same as for designs, it is clear that skip (which corresponds to the design II) is a left identity and fail (which corresponds to the design $\overline{o k}$ ) is a left annihilator w.r.t. composition. Moreover, fail is an identity w.r.t. addition.

Analogously to the case of designs, the normal and feasible prescriptions are the ones for which skip is also a right identity and fail is also a right annihilator w.r.t. composition. The componentwise algebraic transcriptions of these notions are the same as for designs.

Let $\operatorname{NP}(S)$ be the set of normal prescriptions over a semiring $S$. Then we have

Lemma 7.1 The structure $(\mathrm{NP}(S),+, \cdot$, fail, skip) is a weak semiring. 
The identity in algebraic structure is used in the companion paper [4] to give a uniform treatment of normal designs $t \vdash a$ and normal prescriptions $t \Vdash a$ as pairs $(a, t)$ consisting of a transition part $a$ and a termination condition part $t$.

For normal prescriptions we obtain again a simplified composition formula that is isomorphic to $(7)$ :

$$
(a \Vdash b) \cdot(c H d)=(a \wedge \overline{b \cdot \bar{c}}) \Vdash(b \cdot d) .
$$

Finally, we want to relate designs and prescriptions. Following [3], we define

$$
\operatorname{relax}(a \Vdash b)={ }_{d f} a \Vdash(\bar{a}+b)=\left(\begin{array}{cc}
\top & 0 \\
\bar{a} & \bar{a}+b
\end{array}\right) .
$$

Except for the 0 entry this is the representation of a design. We can form a proper design representation by adding one of the matrices

$$
\left(\begin{array}{cc}
0 & \top \\
0 & 0
\end{array}\right)=\overline{o k} \wedge o k^{\prime}=\overline{\text { chaos }} \quad \text { or } \quad\left(\begin{array}{cc}
\top & \top \\
0 & 0
\end{array}\right)=\overline{o k} .
$$

This is summarised in part 1. of the following lemma.

Lemma 7.2 1. $a \vdash b=$ chaos $\rightarrow \operatorname{relax}(a \Vdash b)=o k \rightarrow \operatorname{relax}(a \Vdash b)$. 2. $\operatorname{relax}(a \Vdash b)=(a \vdash b) \wedge$ chaos $=(a \vdash b) \wedge$ ok.

Part 2. follows from part 1. by straightforward Boolean algebra.

\section{Conditions, Tests and Iteration}

As a preparation for our treatment of predicate transformers in the next section, we now show how to algebraically model state predicates that describe sets of states. To keep the framework uniform, state predicates have to be embedded into the general set of predicates or relations. If $M$ is the set of all states then in REL(M) there are three basic methods of representing state predicates, i.e., to characterise subsets $N \subseteq M$, as special predicates or relations:

1. Use predicates that do not depend on the output values of variables, corresponding to right-universal relations $N \times M$. In a semiring with $\top$ they are abstractly characterised as right ideals, i.e., as elements $a$ with $a \cdot \top=a$.

2. Use predicates that do not depend on the input values of variables, corresponding to left-universal relations $M \times N$. In a semiring with $\top$ they are abstractly characterised as left ideals, i.e., as elements $a$ with $\top \cdot a=a$.

3. Use sub-predicates of skip corresponding to partial identity relations of the form $\{(s, s): s \in N\}$. In an idempotent semiring they are abstractly characterised as elements $a$ with $a \leq 1$.

Each of these approaches has its advantages and disadvantages. Classical UTP uses variant 1 , while variant 3 is used in test and modal semirings. Since we are going to import some results from the third framework, we will show some connections between variants 1 and 3 (we do not need variant 2 in the present paper, but the treatment for it would be symmetrical). We only give a summary of the necessary theory; a more thorough treatment can be found in [4]. 
1. A (weak) condition semiring is a pair $(S, \operatorname{cond}(S))$, where $S$ is a (weak) idempotent semiring with a greatest element $\top$ and cond $(S) \subseteq S$ is a Boolean subalgebra of the set of right ideals of $S$ with $0, \top \in \operatorname{cond}(S)$ and such that the join operation in $\operatorname{cond}(S)$ coincides with + and for every element $a \in S$ and every condition $t \in \operatorname{cond}(S)$ the meet $t \wedge a$, called the input restriction of $a$ by $t$, exists and satisfies $(t+u) \wedge a=(t \wedge a)+(u \wedge a)$ as well as $t \wedge(a+b)=t \wedge a+t \wedge b$. We have the correspondences false $\leftrightarrow 0$ and true $\leftrightarrow \top$. The negation of $t$, i.e., its complement relative to $\top$ in $\operatorname{cond}(S)$, is denoted by $\bar{t}$. An example is again $\operatorname{REL}(\mathrm{M})$, with the right-universal relations as conditions.

2. A (weak) test semiring [8] is a pair $(S$, test $(S)$ ), where $S$ is a (weak) idempotent semiring and test $(S) \subseteq[0,1]$ is a Boolean subalgebra of the interval $[0,1]$ of $S$ such that $0,1 \in \operatorname{test}(S)$ and join and meet in test $(S)$ coincide with + and . The negation of test $p$, i.e., its complement relative to 1 in test $(S)$, is denoted by $\neg p$. We have the correspondences false $\leftrightarrow 0$ and true $\leftrightarrow 1$. In a test semiring, for $p \in \operatorname{test}(S)$ and $a \in S$, the products $p \cdot a$ and $a \cdot p$ are the input and output restrictions of $a$ to those pre-/post-states that satisfy $p$. An important example is REL(M) with the partial identities as tests.

We will use the letters $a, b, c, \ldots$ for semiring elements, $p, q, r, \ldots$ for tests and $s, t, u, \ldots$ for conditions. It should be noted that 0 and $T$ are always right (and left) ideals. For 0 this follows from its left annihilation property, while for $\top$ this is property (2).

By associativity of $\cdot$ and property (2) one has $(p \cdot \top) \cdot \top=p \cdot(\top \cdot \top)=p \cdot \top$, i.e., the element $p \cdot T$ is indeed a right ideal. In fact it is easy to show that the right ideals in a semiring $S$ with $\top$ are exactly the products $a \cdot \top$ for $a \in S$.

It can be shown [4] that cond $(S)$ and the set $\operatorname{CS}(S)={ }_{d f}\{t \wedge 1: t \in \operatorname{cond}(S)\}$ of condition subidentities are order-isomorphic. Hence every (weak) condition semiring $S$ can be made into a test (weak) semiring using test $(S)={ }_{d f} \operatorname{CS}(S)$.

To prepare an example of the use of tests we add an operator for finite iteration. A left-inductive Kleene algebra[7] is a structure $\left(S,^{*}\right)$ such that $S$ is an idempotent semiring and the star operation ${ }^{*}: S \rightarrow S$ satisfies, for all $a, b, c \in S$, the left unfold and left induction axioms

$$
1+a \cdot a^{*} \leq a^{*}, \quad b+a \cdot c \leq c \Rightarrow a^{*} b \leq c .
$$

By these axioms, $a^{*} \cdot b$ is the least solution of the fixpoint equation $x=b+a \cdot x$. In particular, the star operator is isotone w.r.t. the natural semiring order. In [4] we have shown that the design and prescription semirings can be made into left-inductive Kleene algebras (and even $\omega$-algebras with infinite iteration $a^{\omega}$ ).

Assume now a test semiring $S$ that also is a left-inductive Kleene algebra. For test $p$ and arbitrary element $a$ one can define the loop "while $p$ do $a$ " in UTP notation as [8]

$$
p * a=_{d f}(p \cdot a)^{*} \cdot \neg p .
$$

The general unfold and induction axioms yield the laws

$$
\neg p+(p \cdot a)^{*} \cdot(p * a) \leq p * a \quad(\text { uf }), \quad \neg p+(p \cdot a) \cdot c \leq c \Rightarrow t * a \leq c \quad \text { (in) } .
$$


With them we show the loop merge law L5 in Section 5.5. of [5]:

$$
(p * a) \cdot((p+q) * a)=((p+q) * a) .
$$

We show this as two inequations. Abbreviate the right hand side by $d$. For $(\leq)$ we have by $\neg p \leq 1$, isotony of star and (uf), unfold

$$
(p \cdot a)^{*} \cdot \neg p \cdot d \leq(p \cdot a)^{*} \cdot d \leq((p+q) \cdot a)^{*} \cdot d \leq d .
$$

The direction $(\geq)$ reduces by (in), Boolean algebra $(p+q=p+\neg p \cdot q)$ and distributivity to the three inequations

$\neg(p+q) \leq(p * a) \cdot d, \quad p \cdot a \cdot(p * a) \cdot d \leq(p * a) \cdot d, \quad \neg p \cdot q \cdot a \cdot(p * a) \cdot d \leq(p * a) \cdot d$.

The first of these holds by (uf) twice, since by Boolean algebra $\neg(p+q)=$ $\neg p \cdot \neg(p+q)$. The second one follows directly from (uf). For the third one we have by the above inequation $(\leq)$ and (uf)

$$
\neg p \cdot q \cdot a \cdot(p * a) \cdot d \leq \neg p \cdot q \cdot a \cdot d \leq(p * a) \cdot(p+q) \cdot a \cdot d \leq(p * a) \cdot d \leq d,
$$

which finishes the proof.

Since we will show below that designs and prescriptions form condition and test semirings, this general result also applies to them, showing the mentioned law L5. Unfortunately, an analogous treatment using conditions instead of tests is a bit more cumbersome.

\section{Domain and Predicate Transformers}

Next we want to characterise the domain of a semiring element $a$, i.e., the set of states from which corresponding output states may be reached under $a$. Again, such sets can be modelled by tests or by conditions.

A simple equational axiomatisation for the case of test semirings has been presented in [2]. We repeat it and give a corresponding axiomatisation for the case of condition semirings in parallel.

The domain operations are

$$
\ulcorner: S \rightarrow \operatorname{test}(S) \quad \pi: S \rightarrow \operatorname{cond}(S)
$$

with the respective axioms

$$
\begin{aligned}
& a \leq\ulcorner a \cdot a \\
& \ulcorner(p \cdot a) \leq p \\
& \ulcorner(a \cdot\ulcorner b) \leq\ulcorner(a \cdot b) \\
& a \leq \pi a \wedge a \\
& \pi(t \wedge a) \leq t \\
& \left.\pi_{(a} \cdot{ }^{\pi} b\right) \leq \pi(a \cdot b)
\end{aligned}
$$

According to [2] $(\operatorname{td} 1) \wedge(\operatorname{td} 2)$ is equivalent to

$$
\ulcorner a \leq p \Leftrightarrow a \leq p \cdot a .
$$


By analogous reasoning we obtain that $(\operatorname{cd} 1) \wedge(\mathrm{cd} 2)$ is equivalent to

$$
\Pi_{a} \leq t \Leftrightarrow a \leq t \wedge a \Leftrightarrow a \leq t
$$

This property has the form of a Galois connection which corresponds to the one for the case of a test semiring with $\top$ (see e.g. [1] and again [2]):

$$
\ulcorner a \leq p \Leftrightarrow a \leq p \cdot \top .
$$

By the Galois connections, the domain operations are unique if they exist.

Moreover, one obtains the following consequences.

\section{Lemma 9.1.}

$$
\begin{aligned}
& \text { 1. }\ulcorner a \leq 0 \Leftrightarrow a \leq 0 \text {, } \\
& \pi_{a} \leq 0 \Leftrightarrow a \leq 0 . \\
& \text { 2. }\ulcorner(a+b)=\ulcorner a+\ulcorner b \text {, } \\
& \pi_{(a+b)}=\pi_{a}+{ }^{\pi} b \text {. } \\
& \text { 3. } a \leq b \Rightarrow \Gamma_{a} \leq \Gamma^{\circ}, \quad a \leq b \Rightarrow \pi_{a} \leq \pi^{\top} b \text {. } \\
& \text { 4. }\ulcorner p=p \text {, } \\
& \pi_{t}=t \\
& \text { 5. } \quad\left\ulcorner\left(\left\ulcorner_{a}\right)=\ulcorner a,\right.\right. \\
& \text { 6. } a=\ulcorner a \cdot a \text {, } \\
& { }^{\pi}\left({ }^{\pi} a\right)=\pi_{a} \text {. } \\
& a=\pi_{a} \wedge a \\
& \text { 7. }\ulcorner(p \cdot a)=p \cdot\ulcorner a \text {, } \\
& \text { 8. }\ulcorner(a \cdot b) \leq\ulcorner(a \cdot\ulcorner b) \text {, } \\
& \text { 9. }\ulcorner(a \cdot \top)=\ulcorner a \text {, } \\
& \text { 10. }\ulcorner(a \cdot b) \leq\ulcorner a \text {, } \\
& \text { 11. }\ulcorner 1=1 \text {, } \\
& \pi(t \wedge a)=t \wedge \pi_{a} . \\
& \left.{ }^{\pi}(a \cdot b) \leq \pi_{\left(a \cdot{ }^{\top}\right.}\right) \text {. } \\
& \pi_{(a \cdot \top)}=\pi_{a} \text {. } \\
& \pi_{(a \cdot b)} \leq \pi_{a} \text {. } \\
& \pi_{1}=\top \text {. }
\end{aligned}
$$

For the proofs in the condition semiring case see [4].

With the help of domain we now define predicate transformers such as wlp and wp that map sets of states to sets of states, both denoted by state predicates. This will allow a more perspicuous representation of the terms involved in the formulas for composition of designs and prescriptions and later the introduction of wlp and wp in the semirings of designs and prescriptions.

The forward modal operators diamond and box are given by

$$
\begin{aligned}
& \langle a\rangle p={ }_{d f}\left\ulcorner(a \cdot p), \quad\left\langle\langle a\rangle t={ }_{d f}{ }^{\top}(a \cdot t),\right.\right. \\
& {[a] p={ }_{d f} \neg\langle a\rangle \neg p, \quad \llbracket a \rrbracket t={ }_{d f} \overline{\langle a\rangle\rangle \bar{t}} .}
\end{aligned}
$$

Thus $\langle a\rangle p /\langle a\rangle\rangle t$ characterise those states for which some $a$-successor state satisfies $p / t$, whereas $[a \rrbracket p / \llbracket a \rrbracket t$ characterise those states for which all a-successor states satisfy $p / t$. The box operators are the abstract counterparts of the wlp operator [13].

From these definitions the following properties are straightforward $[2,4]$.

$$
\begin{aligned}
\langle 0\rangle p & =0, \\
\langle a\rangle(p+q) & =\langle a\rangle p+\langle a\rangle q, \\
\langle a+b\rangle p & =\langle a\rangle p+\langle b\rangle p, \\
\langle p \cdot a\rangle q & =p \cdot\langle a\rangle q, \\
\langle 1\rangle p & =p \\
\langle a \cdot b\rangle p & =\langle a\rangle\langle b\rangle p,
\end{aligned}
$$

$$
\begin{aligned}
\langle 0\rangle\rangle t & =0, \\
\langle a \mid\rangle(t+u) & =\langle\langle a\rangle t+\langle\langle a\rangle\rangle u, \\
\langle\langle a+b\rangle\rangle t & =\langle\langle a\rangle t+\langle b b\rangle t, \\
\langle t \wedge a\rangle u & =t \wedge\langle\langle a\rangle\rangle u, \\
\langle 1\rangle\rangle t & =t, \\
\langle a \cdot b\rangle\rangle t & =\langle\langle a\rangle\langle\langle b\rangle\rangle t .
\end{aligned}
$$


Hence $\langle a\rangle$ and $\langle\langle a\rangle$ are isotone. Moreover, both diamonds are isotone in their first arguments. If the underlying semiring is full, we obtain additionally

$$
\langle a\rangle 0=0 \quad\langle\langle a\rangle 0=0
$$

The box operators enjoy dual laws which we omit, since we will mainly work with diamonds. Because of the importance of modal operators, we call a (weak) test or condition semiring with domain modal.

Now we study the special case of the relation semiring. A (weak) semiring $S$ with $\top$ is ideal-closed, briefly $i d$-closed, if its set $\operatorname{RI}(S)$ of right ideals is a Boolean algebra. The relation semiring REL(M) is id-closed, whereas the semiring of formal languages over an alphabet, under union and concatenation, is not.

We quote the following result from [4]:

Lemma 9.1 For an id-closed weak semiring $S$, the pair $(S, \operatorname{RI}(S))$ can uniquely be made into a weak domain semiring by setting

$$
\pi_{a}={ }_{d f} a \cdot \top .
$$

Hence over an id-closed semiring

$$
\langle a\rangle\rangle t=a \cdot t, \quad \llbracket a \rrbracket t=\overline{a \cdot \bar{t}} .
$$

It should be noted that in [5] the notation $a$ wp $t$ is used for $\llbracket a \rrbracket t$, although really it ought to be $a$ wlp $t$. We will give a proper definition of wp for designs and prescriptions in the next section.

With the above representation of $\llbracket a \rrbracket t$ in id-closed weak semirings we see that the subterm $\overline{b \cdot \bar{c}}$ occurring in the formulas (6) and (10) for composition of designs and prescriptions can be folded into $\llbracket b \rrbracket c$. In the case of a normal design or description, by (7) and (11) the antecedent of the composition therefore simplifies to $a \wedge \llbracket b \rrbracket c$.

\section{Predicate Transformers for Matrices}

Since we have seen that normal designs and prescriptions form weak semirings, we can try to even make them into weak modal semirings. To this end we first need to find out what the potential conditions or tests are in each case. Since, as stated, the condition and test based approaches are isomorphic, we treat only the condition case in the main text, since it is the one used in UTP, and defer the test case to the Appendix.

First we determine the conditions in the design semiring. The greatest (normal) design and also the greatest matrix overall is true. So matrix $A$ is an ideal iff $A \cdot$ true $=A$. Now

$$
\left(\begin{array}{ll}
a & b \\
c & d
\end{array}\right) \cdot\left(\begin{array}{cc}
\top & \top \\
\top & \top
\end{array}\right)=\left(\begin{array}{ll}
(a+b) \cdot \top & (a+b) \cdot \top \\
(c+d) \cdot \top & (c+d) \cdot \top
\end{array}\right)=\left(\begin{array}{ll}
a & b \\
c & d
\end{array}\right)
$$


iff

$$
a=(a+b) \cdot \top=b \quad \wedge \quad c=(c+d) \cdot \top=d .
$$

Hence the ideals are exactly the row-constant matrices with ideals of $S$ as entries. Therefore a normal design $t \vdash a$ is an ideal iff $\bar{t}+a=\bar{t}$, i.e., iff $a \leq \bar{t}$. Such a rowconstant design $\left(\begin{array}{cc}T & T \\ \bar{t} & \bar{t}\end{array}\right)$ has the relative complement $\left(\begin{array}{cc}T & T \\ t & t\end{array}\right)$ within the set of normal designs. Moreover, such a matrix corresponds also to the simpler design $\bar{t} \vdash 0$. The order on the ideals is characterised by $\bar{s} \vdash 0 \leq \bar{t} \vdash 0 \Leftrightarrow s \leq t$. Therefore we have

Theorem 10.1 If $(S, \operatorname{cond}(S))$ is a weak condition semiring, then

$$
(\mathrm{NP}(S),\{\bar{t} \vdash 0: t \in \operatorname{cond}(S)\})
$$

is weak condition semiring. If $\operatorname{cond}(S)=\mathrm{RI}(S)$ then it is id-closed.

If $(S, \operatorname{cond}(S))$ is a modal semiring then $\operatorname{NP}(S)$ is a weak modal semiring with domain operation ${ }^{\pi}(t \vdash a)=\left(t \wedge \overline{\pi_{a}}\right) \vdash 0$.

Proof. The first claim is immediate from the above remarks. For the second claim, we work out what (GCc) means for normal designs: By (4), shunting, lattice algebra, (GCc), 2. and 4. of Lemma 9.1, and Boolean algebra:

$$
\begin{aligned}
& (t \vdash a) \leq(\bar{s} \vdash 0) \\
\Leftrightarrow & \bar{s} \leq t \wedge \bar{s} \wedge a \leq 0 \\
\Leftrightarrow & \bar{t} \leq s \wedge a \leq s \\
\Leftrightarrow & \bar{t}+a \leq s \\
\Leftrightarrow & \pi(\bar{t}+a) \leq s \\
\Leftrightarrow & \bar{t}+\pi a \leq s \\
\Leftrightarrow & \bar{s} \leq t \wedge \bar{\pi}^{\pi} a .
\end{aligned}
$$

Now we check (cd3). By definition of $\pi,(5),(7)$, definition of $\pi$, Boolean algebra and distributivity, since $a \cdot{ }^{\pi} b \leq{ }^{\pi}\left(a \cdot{ }^{\pi} b\right)$ and hence ${ }^{\bar{\pi}\left(a \cdot{ }^{\pi} b\right)} \leq \overline{\left(a \cdot{ }^{\pi} b\right)}$, by modality, definition of $\pi$, and (7):

$$
\begin{aligned}
& \pi_{\left((s \vdash a) \cdot{ }^{\pi}(t \vdash b)\right)} \\
& =\pi^{\pi}((s \vdash a) \cdot(t \wedge \overline{\bar{\pi} b} \vdash 0)) \\
& ={ }_{\pi}^{\pi}\left((s \vdash a) \cdot\left(t \wedge{ }^{\bar{\pi} b} \vdash{ }^{\pi} b\right)\right) \\
& \left.=\left(s \wedge \overline{a \cdot \overline{t \wedge \overline{\pi_{b}}}} \vdash a \cdot{ }^{\pi} b\right)\right) \\
& =\left(s \wedge \overline{a \cdot \overline{t \wedge \overline{\bar{\pi}}}} \wedge \overline{\pi^{\pi}\left(a \cdot{ }^{\pi} b\right)}\right) \vdash 0 \\
& =\left(s \wedge \overline{a \cdot \bar{t}} \wedge \overline{a \cdot{ }^{\pi} b} \wedge \overline{\pi\left(a \cdot{ }^{\pi} b\right)}\right) \vdash 0 \\
& =\left(s \wedge \overline{a \cdot \bar{t}} \wedge \overline{\left.\pi^{\left(a \cdot{ }^{\pi} b\right)}\right)} \vdash 0\right. \\
& =(s \wedge \overline{a \cdot \bar{t}} \wedge \bar{\pi}(a \cdot b)) \vdash 0 \\
& =\pi_{(s \wedge \overline{a \cdot \bar{t}} \vdash a \cdot b)} \\
& =\pi((s \vdash a) \cdot(t \vdash b)) \text {. }
\end{aligned}
$$


Let us work out the box operator for the case of a modal underlying $S$ : By the definitions, complement of ideal, (5), (7), definition, since $a \cdot \bar{s} \leq{ }^{\pi}(a \cdot \bar{s})$, hence $\overline{\bar{\pi}(a \cdot \bar{s})} \leq \overline{a \cdot \bar{s}}$, definition,

$$
\begin{aligned}
& \frac{\llbracket t \vdash a \rrbracket(\bar{s} \vdash 0)}{\pi((t \vdash a) \cdot \overline{\bar{s} \vdash 0)})} \\
= & \frac{((t \vdash a) \cdot(s \vdash 0))}{\pi} \\
= & \frac{((t \vdash a) \cdot(s \vdash \bar{s}))}{\pi(t \wedge \overline{a \cdot \bar{s}}) \vdash a \cdot \bar{s})} \\
= & \overline{\left(t \wedge \overline{a \cdot \bar{s}} \wedge^{\bar{\pi}(a \cdot \bar{s})} \vdash 0\right.} \\
= & \frac{t \wedge \bar{\pi}(a \cdot \bar{s})}{\pi}-0 \\
= & \overline{t \wedge \llbracket a \rrbracket s} \vdash 0 .
\end{aligned}
$$

This corresponds precisely to the definition of the wp operator in [13]. That wp is just the wlp of another semiring seems first to have been noted in [10] for a test-based approach.

For the case of prescriptions things work much in the same way. Again we have $t H a \leq$ skip $\Leftrightarrow t=\top \wedge a \leq 1$. The ideals have to satisfy $(t H a) \cdot$ chaos $=$ $(t \Vdash a)$, which works out to

$$
a \cdot \top=a \wedge a \leq \bar{t}
$$

Hence we can choose the sets of tests and conditions as in the case of designs and obtain a (non-id-closed) weak modal semiring $\mathrm{NP}(S)$.

\section{Matrices of Predicate Transformers}

In this section we show that the matrix calculus can be extended to predicate transformers, which will allow a lifting of the results of Sections 5 and 7 to predicate transformer algebras. Doing this, we obtain the simplified composition formulas for normal designs with less complicated calculations, while at the same time removing the need for the underlying semiring to be id-closed.

First we show that the diamond operators over a condition semiring form a condition semiring again.

Lemma 11.1 Set, for $U \subseteq S$ in a weak modal condition semiring $(S, \operatorname{cond}(S))$,

$$
\left.\langle U\rangle={ }_{d f}\{\langle a\rangle\rangle: a \in U\right\} .
$$

1. The structure $\left\langle\langle S\rangle={ }_{d f}(\langle S\rangle\rangle,+, \circ,\langle\langle 0\rangle,\langle\langle 1\rangle)\right.$ is a (weak) semiring with greatest element $\langle\langle T\rangle$ under the operations

$$
\left\langle\langle a\rangle+\left\langle\langle b\rangle={ }_{d f}\left\langle\langle a+b\rangle, \quad\langle\langle a\rangle\rangle\left\langle\langle b\rangle={ }_{d f}\langle\langle a \cdot b\rangle .\right.\right.\right.\right.
$$


2. For $s, t \in \operatorname{cond}(S)$ we have $\langle t \wedge u\rangle=\langle\langle t\rangle \wedge\langle\langle u\rangle$, where the meet of diamonds is defined pointwise.

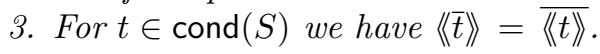

4. $\{\langle a\rangle: a \in \operatorname{RI}(S)\} \subseteq \operatorname{RI}(\langle S\rangle)$.

Proof. 1. This is immediate from the diamond properties.

2. We calculate, for $u \in \operatorname{cond}(S)$, using Lemma 9.1.7,

$$
\langle s \wedge t\rangle\rangle u=s \wedge t \wedge\langle T T\rangle u=s \wedge\langle\langle T\rangle u \wedge t \wedge\langle\langle T\rangle u=\langle\langle s\rangle u \wedge\langle\langle t\rangle u .
$$

3. First, $\langle\langle t\rangle+\langle\bar{t}\rangle\rangle=\langle t+\bar{t}\rangle\rangle=\langle\langle T\rangle$. Second, by 2 ., $\langle\langle t\rangle\rangle \wedge\langle\bar{t}\rangle\rangle=\langle t \wedge \bar{t}\rangle\rangle=\langle\langle 0\rangle$.

The laws of involution and de Morgan are also easily checked.

4. $a \in \operatorname{RI}(S) \Leftrightarrow a=a \cdot \top \Rightarrow\langle\langle a\rangle=\langle\langle a \cdot T\rangle=\langle\langle a\rangle \circ\langle\langle\top\rangle$.

In the remainder we will mostly omit the composition operator $\circ$.

We now define a property that relaxes the one of id-closedness (see Section 9). We call a (weak) modal condition semiring $S$ Tarskian if it satisfies

$$
\langle T\rangle u=T \Leftarrow u \in \operatorname{cond}(S) \backslash\{0\} .
$$

By Lemma 9.1.9, (MTARt) is equivalent to

$$
\pi(\top \cdot u \cdot \top)=\top \Leftarrow u \in \operatorname{cond}(S) \backslash\{0\} .
$$

This is a modal analogue of the Tarski rule $T \cdot a \cdot T=T \Leftarrow a \neq 0$ of the relational calculus, whence our terminology.

Property (MTARt) holds in REL(M), but also in many other semirings that, contrary to REL(M), are not id-closed, e.g. in the semirings of languages of finite and infinite words under concatenation and under fusion product.

We obtain another useful equivalent characterisation:

Lemma 11.2 $S$ is Tarskian iff $\langle t\rangle u=t$ for all $t, u \in \operatorname{cond}(S)$ with $u \neq 0$.

Proof. $(\Rightarrow)\langle t\rangle u=t \wedge\langle\top \top\rangle u=t \wedge \top=t$.

$(\Leftarrow)$ Set $t=T$.

Lemma 11.3 Assume a Tarskian modal condition semiring $(S, \operatorname{cond}(S))$.

1. For all $a \in S$ we have $\left.\langle\langle a\rangle\langle T\rangle\rangle=\left\langle{ }^{\top} a\right\rangle\right\rangle$.

2. $\operatorname{RI}(\langle S\rangle)=\{\langle t\rangle\rangle: t \in \operatorname{cond}(S)\}$.

3. $(\langle S\rangle\rangle, \operatorname{RI}(\langle S\rangle\rangle))$ is an id-closed and Tarskian modal condition semiring with $\pi\langle a\rangle=\left\langle\|^{\pi} a\right\rangle$.

Proof. 1. Since we assume a semiring and not just a weak semiring,

$$
\langle a\rangle\rangle\langle T\rangle\rangle=\left\langle\langle a\rangle 0=0=\left\langle{ }^{\top} a\right\rangle\right\rangle 0 .
$$

For $u \neq 0$ we calculate, using Lemma 11.2,

$$
\langle a\rangle\rangle\langle T\rangle u=\langle a\rangle\rangle \top=\pi^{\pi} a=\left\langle{ }^{\top} a\right\rangle u .
$$


2. By 1. we have $\langle a \mid\rangle \in \operatorname{RI}(\langle S\rangle) \Leftrightarrow\left\langle\langle a\rangle=\left\langle\langle a\rangle\left\langle\langle T\rangle \Leftrightarrow\left\langle\langle a\rangle=\left\langle{ }^{\top} a\right\rangle\right.\right.\right.\right.$.

3. Immediate from 2.,1. and Lemma 9.1.

Given these results we can now use

$$
\left(\begin{array}{cc}
\langle T T\rangle & \langle\langle T\rangle \\
\langle\bar{t}\rangle & \langle\bar{t}+a\rangle
\end{array}\right) \quad \text { and } \quad\left(\begin{array}{cc}
\langle T T\rangle & \langle 00\rangle \\
\langle\bar{t}\rangle & \langle a\rangle\rangle
\end{array}\right)
$$

as predicate transformer representations of normal design $t \vdash a$ and prescription $t H a$, resp., over a Tarskian modal semiring. For the lower left corner element of both $(t \vdash a) \cdot(u \vdash b)$ and $(t \Vdash a) \cdot(u \Vdash b)$ we obtain

$$
\langle\langle\bar{t}\rangle\langle\langle T\rangle+\langle\langle a\rangle\langle\bar{u}\rangle\rangle=\langle\bar{t}\rangle+\langle\langle a\rangle\rangle\langle\bar{u}\rangle\rangle=\overline{\langle t\rangle\rangle \wedge \llbracket\langle a \mid\rangle \rrbracket\langle u\rangle,}
$$

so that things work now smoothly even for non-id-closed underlying semiring $S$.

\section{Conclusion and Outlook}

The matrix calculus has proved to be a convenient vehicle for reasoning about general UTP predicates as well as designs and prescriptions. Their modal semiring structure allows re-use of the large existing body of results about Kleene/ $\omega$ algebra with tests and modal Kleene/ $\omega$ algebra. Recently it has also been shown [6] that designs and prescriptions form a demonic refinement algebra in the sense of von Wright [14], so that that framework can be re-used, too.

It remains to be seen whether a similar approach can be followed when further observation variables are added.

Acknowledgements: I am grateful to Walter Guttmann, Peter Höfner, Kim Solin and the anonymous referees for helpful discussions and remarks.

\section{References}

1. C.J. Aarts: Galois connections presented calculationally. M.S. thesis, Department of Mathematics and Computing Science, Eindhoven University of Technology 1992

2. J. Desharnais, B. Möller, G. Struth: Kleene algebra with domain. ACM TOCL 2006 (to appear)

3. S. Dunne: Recasting Hoare and He's unifying theory of programs in the context of general correctness. In A. Butterfield, G. Strong, C. Pahl (eds.): 5th Irish Workshop on Formal Methods. EWiC, The British Computer Society, 2001

4. W. Guttmann, B. Möller: Modal design algebra. Institut für Informatik, Universität Augsburg, Report 2005-15. Revised version in S. Dunne, B. Stoddart(eds.): UTP 2006 - Proc. First International Symposium on Unifying Theories of Programming, Walworth Castle, County Durham, UK, 5-7 Feb. 2006. LNCS 4010. Springer 2006, 236-256 (in press)

5. C.A.R. Hoare, J. He: Unifying theories of programming. Prentice Hall 1998

6. P. Höfner, B. Möller, K. Solin: Omega Algebra, Demonic Refinement Algebra and Commands. Institute of Computer Science, University of Augsburg, Technical Report 2006-11, March 2006 
7. D. Kozen: A completeness theorem for Kleene algebras and the algebra of regular events. Information and Computation 110:2, 366-390 (1994)

8. D. Kozen: Kleene algebra with tests. ACM TOPLAS 19:427-443 (1997)

9. B. Möller, G. Struth: Algebras of modal operators and partial correctness. Theoretical Computer Science 351, 221-239 (2006)

10. B. Möller, G. Struth: wp is wlp. In W. MacCaull, M. Winter and I. Düntsch (eds.): Relational Methods in Computer Science. LNCS 3929. Springer 2006, 200-211 (in press)

11. C. Morgan: Data Refinement by Miracles. Inf. Process. Lett. 26, 243-246 (1988)

12. J.M. Morris, Laws of data refinement, Acta Informatica (26), 287-308 (1989)

13. G. Nelson: A generalization of Dijkstra's calculus. ACM TOPLAS 11, 517-561 (1989)

14. J.von Wright: Towards a refinement algebra. Science of Computer Programming $51,23-45(2004)$

\section{A Appendix: Test-Based Predicate Transformers}

First we determine the tests in the semiring of designs. The multiplicative identity is $\mathbb{I}=\top \vdash 1$ and by (4) we obtain

$$
t \vdash a \leq \mathbb{I} \Leftrightarrow(\top \leq t) \wedge(\top \wedge a \leq 1) .
$$

So the subidentities are of the form $\top \vdash p$ with $p \leq 1$. Moreover,

$$
(\top \vdash p)+(\top \vdash q)=(\top \vdash p+q)
$$

and, by (6),

$$
(\top \vdash p) \cdot(\top \vdash q)=(\top \vdash p \cdot q) .
$$

Hence, if $p \leq 1$ has the relative complement $q \leq 1$ w.r.t. 1 then $\top \vdash q$ is the complement of $\top \vdash p$ relative to $\mathbb{I}$. This shows

Lemma A.1 If $(S$, test $(S))$ is a weak test semiring, then so is

$$
(\mathrm{ND}(S),\{\top \vdash p: p \in \operatorname{test}(S)\}) \text {. }
$$

We use the characterisation (12) of domain to find out whether we can even make $\mathrm{ND}(S)$ into a weak domain semiring if $S$ is one: By (7) and lattice algebra, (4), shunting, lattice algebra, distributivity and (12), and lattice algebra and additivity of domain:

$$
\begin{aligned}
& t \vdash a \leq(\top \vdash p) \cdot(t \vdash a) \\
\Leftrightarrow & t \vdash a \leq \overline{p \cdot \bar{t}} \vdash p \cdot a \\
\Leftrightarrow & \bar{p} \cdot \bar{t} \leq t \wedge \overline{p \cdot \bar{t}} \wedge a \leq p \cdot a \\
\Leftrightarrow & \bar{t} \leq p \cdot \bar{t} \wedge a \leq p \cdot \bar{t}+p \cdot a \\
\Leftrightarrow & \bar{t} \leq p \cdot \bar{t} \wedge \bar{t}+a \leq p \cdot \bar{t}+p \cdot a \\
\Leftrightarrow & \bar{t} \leq p \wedge(\bar{t}+a) \leq p \\
\Leftrightarrow & \ulcorner(\bar{t}+a) \leq p .
\end{aligned}
$$


So setting

$$
\left\ulcorner(t \vdash a)={ }_{d f} \top \vdash\ulcorner(\bar{t}+a)\right.
$$

we satisfy (td1) and (td2); a straightforward calculation shows that also (td3) holds. Altogether we have shown

Theorem A.2 If $(S$, test $(S),\ulcorner)$ is a weak modal semiring, then $\operatorname{ND}(S)$ can be made into a weak modal semiring.

For the case of prescriptions things work much in the same way. Again we have $t \Vdash a \leq$ skip $\Leftrightarrow t=\top \wedge a \leq 1$. Hence we can choose the set of tests as in the case of designs and obtain a test-based weak modal semiring $\operatorname{NP}(S)$.

Next, as in the case of conditions, we investigate the semiring structure of the test-based diamond operators.

Lemma A.3 Consider a (weak) modal test semiring $(S$, test $(S))$ and set, for $U \subseteq S$,

$$
\langle U\rangle={ }_{d f}\{\langle a\rangle: a \in U\} .
$$

1. The structure $\langle S\rangle={ }_{d f}(\langle S\rangle,+, \circ,\langle 0\rangle,\langle 1\rangle)$ is a (weak) semiring with greatest element $\langle\top\rangle$ under the operations

$$
\langle a\rangle+\langle b\rangle={ }_{d f}\langle a+b\rangle, \quad\langle a\rangle \circ\langle b\rangle={ }_{d f}\langle a \cdot b\rangle .
$$

2. For $p, q \in \operatorname{test}(S)$ we have $\langle p \cdot q\rangle=\langle p\rangle \wedge\langle q\rangle$.

3. For $a \in S$ we obtain $\langle a\rangle \leq\langle 1\rangle \Leftrightarrow\langle a\rangle=\langle\ulcorner a\rangle$.

4. For $p \in \operatorname{test}(S)$ one has $\langle\neg p\rangle=\neg\langle p\rangle$.

5. $\{\langle a\rangle: a \in \operatorname{RI}(S)\} \subseteq \operatorname{RI}(\langle S\rangle)$.

Proof. 1. This is shown in [9].

2. We calculate, for $r \in \operatorname{test}(S)$,

$$
\langle p \cdot q\rangle r=p \cdot q \cdot r=p \cdot r \cdot q \cdot r=\langle p\rangle r \wedge\langle q\rangle r .
$$

3. By isotony of the diamond we only need to show $(\Rightarrow)$. Consider an arbitrary $p \in \operatorname{test}(S)$.

$$
\begin{aligned}
\langle\ulcorner a\rangle p & =\ulcorner a \cdot p=p \cdot\ulcorner a=p \cdot\ulcorner(a \cdot p+a \cdot \neg p)=p \cdot\ulcorner(a \cdot p)+p \cdot\ulcorner(a \cdot \neg p) \\
& =p \cdot\langle a\rangle p+p \cdot\langle a\rangle \neg p=\langle a\rangle p+0=\langle a\rangle p,
\end{aligned}
$$

since by assumption $\langle a\rangle p \leq p$ and $\langle a\rangle \neg p \leq \neg p$.

4. First, $\langle p\rangle+\langle\neg p\rangle=\langle p+\neg p\rangle=\langle 1\rangle$. Second, by 2., $\langle p\rangle \wedge\langle\neg p\rangle=\langle p \cdot \neg p\rangle=\langle 0\rangle$.

The laws of involution and de Morgan are also easily checked.

5. $a \in \operatorname{RI}(S) \Leftrightarrow a=a \cdot \top \Rightarrow\langle a\rangle=\langle a \cdot \top\rangle=\langle a\rangle \circ\langle\top\rangle$.

In the remainder we will again mostly omit the composition operator. Lemma A.3.3 allows us to define domain on diamonds:

Theorem A.4 Setting $\left\ulcorner\langle a\rangle={ }_{d f}\langle\ulcorner a\rangle\right.$ makes $(\langle S\rangle,\langle\operatorname{test}(S)\rangle)$ into a modal test semiring. 
Proof. By the previous lemma $\langle\operatorname{test}(S)\rangle$ is a test algebra. So we only need to check the domain axioms.

$(\operatorname{cd} 1)\lceil\langle a\rangle \circ\langle a\rangle=\langle\ulcorner a\rangle \circ\langle a\rangle=\langle\ulcorner a \cdot a\rangle=\langle a\rangle$.

$(\mathrm{cd} 2)\ulcorner(\langle p\rangle \circ\langle a\rangle)=\langle p \cdot a\rangle=\langle\ulcorner(p \cdot a)\rangle \leq\langle p\rangle$.

(cd3) $\ulcorner(\langle a\rangle \circ\langle b\rangle)=\ulcorner\langle a \cdot b\rangle=\langle\ulcorner(a \cdot b)\rangle=\langle\ulcorner(a \cdot\ulcorner b)\rangle=\ulcorner(\langle a\rangle \circ\langle\ulcorner b\rangle)=\ulcorner(\langle a\rangle \circ\ulcorner\langle b\rangle)$.

We conclude by relating the test and condition based approaches. A (weak) modal test semiring $S$ is Tarskian if satisfies

$$
\langle\top\rangle q=1 \Leftarrow q \in \operatorname{test}(S) \backslash\{0\},
$$

or, equivalently,

$$
\ulcorner(\top \cdot q \cdot \top)=1 \Leftarrow q \in \operatorname{test}(S) \backslash\{0\} .
$$

We define the set of test ideals of $S$ as $\operatorname{TI}(S)=_{d f}\{p \cdot \top: p \in \operatorname{test}(S)\}$. From [2] we know that $\overline{p \cdot \top}=\neg p \cdot \top$ and $p \cdot \top \leq q \cdot \top \Leftrightarrow p \leq q$. Using test ideals we obtain another characterisation of the Tarskian property:

Lemma A.5 $S$ is Tarskian iff $\langle p \cdot T\rangle q=p$ for all $p, q \in \operatorname{test}(S)$ with $q \neq 0$.

Proof. $(\Rightarrow)\langle p \cdot \top\rangle q=\langle p\rangle\langle\top\rangle q=\langle p\rangle 1=p$.

$(\Leftarrow)$ Set $p=1$.

Lemma A.6 Assume a Tarskian modal test semiring $(S$, test $(S))$.

1. For all $a \in S$ we have $\langle a\rangle\langle\top\rangle=\langle\ulcorner a \cdot \top\rangle$.

2. $\operatorname{RI}(\langle S\rangle)=\langle\operatorname{TI}(S)\rangle$.

3. $(\langle S\rangle,\langle\operatorname{TI}(S)\rangle)$ is an id-closed and Tarskian modal condition semiring with $\pi\langle a\rangle=\langle[\ulcorner a\rangle$.

Proof. 1. Since we assume a semiring and not just a weak semiring,

$$
\langle a\rangle\langle T\rangle 0=\langle a\rangle 0=0=\left\langle{ }^{a} a\right\rangle 0 .
$$

For $q \neq 0$ we calculate, using Lemma A.5,

$$
\langle a\rangle\langle\top\rangle q=\langle a\rangle 1=\ulcorner a=\langle\ulcorner a \cdot \top\rangle q .
$$

2. By 1., $\langle a\rangle \in \operatorname{RI}(\langle S\rangle) \Leftrightarrow\langle a\rangle=\langle a\rangle\langle T\rangle \Leftrightarrow\langle a\rangle=\langle\ulcorner a \cdot T\rangle$.

3. Immediate from 2.,1. and Lemma 9.1.

Therefore we can, over a Tarskian modal test semiring, represent normal designs and prescriptions also in the forms

$$
p \vdash a=_{d f}\left(\begin{array}{cc}
\langle\top\rangle & \langle T\rangle \\
\langle\neg p \cdot T\rangle & \langle\neg p \cdot T+a\rangle
\end{array}\right) \quad \text { and } \quad p H a=_{d f}\left(\begin{array}{cc}
\langle\top\rangle & \langle 0\rangle \\
\langle\neg p \cdot T\rangle & \langle a\rangle
\end{array}\right)
$$

For the lower left corner element of both $(p \vdash a) \cdot(q \vdash b)$ and $(p \Vdash a) \cdot(q \Vdash b)$ we now obtain, with the test ideal $t=_{d f} p \cdot T$ and arbitrary test ideal $u$,

$$
\langle\bar{t}\rangle+\langle a\rangle\langle\bar{u}\rangle=\neg(\langle t\rangle \wedge[\langle a\rangle]\langle u\rangle),
$$

and things work again smoothly even for non-id-closed underlying semiring $S$. 